\title{
因HAD
}

ISSN-L: 2530-5115

DOI: http://doi.org/10.22585/hospdomic.v2i1.21

\section{Ingresos en hospitalización a domicilio directamente desde el servicio de urgencias: una alternativa eficaz y segura}

\section{Admissions in hospital at home directly from the emergency department: an effective and safe alternative}

Leticia María Dopico Santamariña

Complejo Hospitalario Universitario A Coruña, Unidad de Hospitalización a Domicilio, A Coruña, España.

Correspondencia/Correspondence

Leticia María Dopico Santamariña

leticiadopico@gmail.com

Recibido/Received

13.06.2017

Aceptado/Accepted

08.11 .2018
Conflicto de Intereses/Competing interest La autora declara no tener ningún conflicto de intereses.

Agradecimientos/Acknowledgments

Agradecimientos al Dr. Hassan Abdulkadir Asaad por su colaboración en el proceso de recogida de datos y al Dr. Fernando Lamelo Alfonsín por la aportación de ideas durante la realización del presente estudio. 


\section{RESUMEN}

Objetivo: Analizar el perfil de pacientes con patología infecciosa que ingresan en $\mathrm{HaD}$ tras captación en Urgencias, soporte y calidad asistencial.

Método: Estudio observacional retrospectivo. Variables: características del paciente, infección, tratamiento, parámetros de hospitalización e indicadores de calidad.

Resultados: Incluidos 255 pacientes con edad media 71 años (48.63\% varones; $51.37 \%$ mujeres) e índice medio de Charlson 4.91. Diagnósticos más frecuentes: infección urinaria (25.10\%) y respiratoria $(24.71 \%)$, sin identificación microbiológica en $62.75 \%$. Aislamiento más frecuente: E.coli (21.32\%) documentándose 10.20\% bacteriemia y $14.51 \%$ criterios de sepsis. Todos necesitaron antibioterapia parenteral con media 6.5 días, siendo más empleados carbapenems (34.31\%). Estancia media en Urgencias 21 horas y en HaD 7 días. Se registraron $9.41 \%$ de complicaciones obteniéndose tasa de reingreso $2.75 \%$.

Conclusiones: Tras estancia corta en Urgencias es posible el manejo eficaz de infecciones con criterios variables de gravedad y alta comorbilidad, dando el soporte necesario desde HaD, sin aumentar complicaciones ni reingresos.

Palabras clave: hospitalización a domicilio; urgencias; infección; resistencia antimicrobiana; antibioterapia parenteral; terapia de infusión ambulatoria.

\section{ABSTRACT}

Objective: To analyze the profile of patients with infectious pathology who enter hospital at home after collection in the emergency department, support and quality of care.

Method: Retrospective observational study. Variables: characteristics of the patient, type of infection, treatment, hospitalization parameters and quality indicators.

Results: Including 255 patients with mean age 71 years (48.63\% male, $51.37 \%$ female) and average Charlson index 4.91. Most frequent diagnoses: urinary tract infection (25.10\%) and respiratory infection $(24.71 \%)$, without microbiological identification in $62.75 \%$. Most frequent isolation: E. coli (21.32\%), with bacteremia being documented in $10.20 \%$ and criteria of sepsis in $14.51 \%$. All of them required parenteral antibiotic therapy with average 6.5 days, with carbapenems being the most used (34.31\%). Average stay in emergency department 21 hours and in hospital at home 7 days. There were $9.41 \%$ of complications, obtaining a reentry rate of $2.75 \%$.

Conclusions: After a short stay in emergency department, it's possible to manage infections with variable criteria of severity and high comorbidity, giving the necessary support from hospital at home, without increasing complications or readmissions.

Keywords: hospital-based home care; emergency department; infection; antimicrobial drug resistance; intravenous antibiotic; outpatient infusion therapy. 


\section{INTRODUCCIÓN}

Las unidades de hospitalización a domicilio $(\mathrm{HaD})$ constituyen una alternativa asistencial en aquellos pacientes que precisan ingreso hospitalario por diversas causas y cumplen una serie de criterios que los convierten en candidatos válidos para recibir dicha atención en su domicilio (1). Se trata, por tanto, de proporcionar asistencia sanitaria especializada en el hogar del paciente cuya situación clínica hace que se requieran tratamientos de rango hospitalario que, de otro modo, implicarían su ingreso en una planta de hospitalización convencional (2).

Desde el año 1981, este modelo asistencial se ha implantado progresivamente en nuestro país destacando una gran heterogeneidad entre las distintas unidades, tanto a nivel organizativo como en lo referente a su actividad, sin olvidar las desigualdades en su distribución geográfica (3).

La unidad de hospitalización a domicilio del Complejo Hospitalario Universitario de A Coruña fue creada en el año 1987 y, a lo largo de los últimos años, ha ido adaptando su modelo organizativo a las necesidades surgidas como consecuencia del envejecimiento poblacional y la alta incidencia de patología infecciosa, en muchos casos, debido a gérmenes multirresistentes. De la creciente demanda de atención en el servicio de urgencias (4) y el aumento de ingresos hospitalarios caracterizados por un perfil de pacientes con un alto grado de comorbilidad, nace, en Diciembre de 2013, un proyecto estratégico de captación activa de pacientes en el servicio de urgencias. Dichos pacientes son ingresados directamente en la unidad de hospitalización a domicilio, sin previo paso por planta de hospitalización convencional ni unidades de corta estancia médica (5).

Para la puesta en marcha del proceso de captación, un médico de la unidad realiza diariamente la revisión de las historias clínicas de los pacientes, que se encuentran en el servicio de urgencias en espera de ser ingresados en una planta de hospitalización; y aquellos en observación pendientes de decidir su destino según la evolución. Tras dicha revisión, se realiza una selección de posibles candidatos y su posterior valoración en el servicio de urgencias, en base a criterios médicos (estabilidad clínica, diagnóstico establecido y posibilidad de realizar el tratamiento en el domicilio) y criterios sociodemográficos (domicilio en el área de cobertura de $\mathrm{HaD}$, medios de contacto, existencia de un cuidador principal que se responsabilice del paciente y aceptación voluntaria del ingreso en la unidad).

De acuerdo a lo expuesto, se plantea un estudio con el objetivo de analizar el perfil de pacientes que ingresan en nuestra unidad con diagnóstico de patología infecciosa tras captación activa en el servicio de urgencias, su manejo, el soporte requerido y las implicaciones en la calidad asistencial.

\section{MATERIAL Y MÉTODOS}

Se diseñó un estudio observacional descriptivo retrospectivo incluyendo una muestra de 255 pacientes cuyo criterio de inclusión se basó en haber ingresado en la unidad de HaD del Complejo Hospitalario Universitario A Coruña, procedentes del servicio de urgencias con diagnóstico de enfermedad infecciosa entre el 1 de junio de 2014 y el 30 de junio de 2015.

Las variables estudiadas se dividieron en los siguientes grupos: características del paciente (edad, sexo, índice de comorbilidad de Charlson); tipo de infección (diagnóstico, identificación microbiológica, germen aislado, bacteriemia documentada, criterios de sepsis/síndrome de respuesta inflamatoria sistémica (SIRS) según el American College of Chest Psysicians y la Society of Critical Medicine); tratamiento parenteral (tipo de antibiótico, duración del tratamiento, terapia secuencial, causas de no realizar tratamiento secuencial); parámetros de hospitalización (estancia en urgen- 
cias, estancia en $\mathrm{HaD}$ ) e indicadores de calidad (complicaciones, reingreso durante la estancia en $\mathrm{HaD}$, reingreso hospitalario por el mismo motivo durante el primer mes tras el alta).

La recogida de datos se realizó mediante el acceso a la historia clínica electrónica del Servicio Gallego de Salud (programa IANUS) con registro posterior en hoja de cálculo tipo Excel. Las variables cuantitativas se expresaron mediante medidas de centralización y dispersión, mientras que las cualitativas fueron representadas por sus valores absoluto y relativo. El análisis estadístico se realizó mediante el Programa de Análisis Epidemiológico de Datos EPIDAT, versión 4.2.

\section{RESULTADOS}

Durante el período estudiado, ingresaron en nuestra unidad 382 pacientes procedentes del servicio de urgencias, de los cuales 255 (66.75\%) presentaron patología infecciosa como motivo de ingreso siendo incluidos en el estudio. La edad media de los pacientes fue de 71 años con una desviación estándar de 19 años. Por sexos, la muestra estuvo formada por 124 varones (48.63\%) y 131 mujeres (51.37\%). El índice medio de comorbilidad de Charlson fue de 4.91 con una desviación estándar de 2.97.

Respecto al tipo de infección, el diagnóstico más frecuente fue la infección urinaria (25.10\%), seguida de infección respiratoria (24.71\%) y neumonía (21.96\%). El aislamiento microbiológico más frecuente fue E. coli con un valor absoluto de 62 (24.03\%), de los cuales, 7 (11.29\% del total de E. coli y $2.71 \%$ del total de aislamientos) fueron productores de betalactamasas de espectro extendido (BLEE). Si bien, en 160 casos (62.75\%) no se dispuso de identificación microbiológica, se documentó bacteriemia en 26 (10.20\%) y criterios de sepsis/SIRS en 37 (14.51\%).

Todos los pacientes recibieron tratamiento antibiótico parenteral siendo los carbapenems los más empleados (34.31\%), seguidos de cefalosporinas (33.21\%) y quinolonas (28.10\%). La duración media del tratamiento parenteral fue de 6.5 días (mediana: 5) con una desviación estándar de 4.68 días. En 171 pacientes (67.06\%) se realizó tratamiento secuencial con antibiótico por vía oral, restando 84 que no lo recibieron por diversas razones: 51 habían completado la pauta de tratamiento por vía intravenosa (60.71\%), 11 presentaban dificultad para la deglución (13.10\%) y en 6 casos no se disponía de alternativa antimicrobiana por vía oral (13.10\%).

Respecto a los parámetros de hospitalización, la estancia media en el servicio de urgencias fue de 20.55 horas (mediana: 21 horas) con una desviación estándar de 10.24 horas. En HaD, la estancia media fue de 8.77 días (mediana: 7 días) con una desviación estándar de 7.38 días.

Por último, 231 pacientes (90.59\%) finalizaron su ingreso en HaD sin presentar complicaciones frente a 24 (9.41\%) que sí las presentaron siendo en su mayoría alteraciones analíticas reversibles. $\mathrm{La}$ tasa de reingreso hospitalario durante el ingreso en $\mathrm{HaD}$ fue del $2.75 \%$ (7 pacientes) con una tasa de reingreso en el primer mes tras el alta del 7.06\% (18 pacientes).

\section{DISCUSIÓN}

La patología infecciosa es la primera causa de ingreso hospitalario en los pacientes que acuden al servicio de urgencias, ya sea de forma aguda o crónica agudizada. Frecuentemente, se trata de pacientes de edad avanzada con alta comorbilidad y riesgo de complicaciones (6), presentando criterios variables de gravedad incluyendo bacteriemia, sepsis y síndrome de respuesta inflamatoria sistémica. 
Esto implica la necesidad de dar soporte mediante la administración de antibioterapia parenteral en el domicilio, para la cual han de tenerse en cuenta las propiedades farmacocinéticas y farmacodinámicas del antibiótico. En la medida de lo posible, se elegirán fármacos que requieran administración endovenosa una vez al día con el fin de disminuir la manipulación del catéter y facilitar la asistencia en el horario de cobertura de la unidad. Si bien, existe la posibilidad de la utilización de bombas elastoméricas o electromecánicas en casos seleccionados, debiendo considerar la estabilidad del antibiótico a temperatura ambiente. Este tipo de terapias han sido estudiadas a lo largo de las últimas décadas, habiendo demostrado seguridad y eficacia comparable con la administración en el medio hospitalario $(7,8)$.

Por otro lado, en los últimos años, se ha incrementado la incidencia de infecciones causadas por gérmenes multirresistentes, lo cual dificulta la elección del tratamiento antimicrobiano empírico pudiendo ensombrecer el pronóstico a largo plazo. Cabe mencionar que en nuestro estudio no se dispuso de identificación microbiológica en más de la mitad de los casos.

Con una estancia media en urgencias inferior a 21 horas, hemos comprobado que es posible el manejo eficaz de estos pacientes proporcionando el soporte necesario desde una unidad de hospitalización a domicilio, sin detectarse un aumento de las complicaciones ni en la tasa de reingreso hospitalario, manteniendo una estancia media en la unidad equiparable a otros sistemas de hospitalización convencional (9).

Como limitación en este estudio hay que destacar la obtención de datos en relación con la admisión del paciente en el servicio de urgencias y, posteriormente, en la unidad dado que el registro horario es realizado por personal administrativo una vez reciben el historial en soporte papel del paciente. Por tanto, es probable que la estancia media en urgencias sea incluso menor al resultado obtenido.

Estos hallazgos refuerzan a las unidades de $\mathrm{HaD}$ como una alternativa válida (10) de ingreso desde la puerta de entrada al sistema hospitalario, manteniendo la calidad asistencial y contribuyendo a una mejor gestión de las camas hospitalarias así como a la desaturación del servicio de urgencias, especialmente, en épocas de mayor demanda asistencial coincidiendo con el aumento de patología infecciosa estacionaria.

En el futuro, cabría valorar la posibilidad de evaluar la adecuación de los tratamientos antibióticos empíricos instaurados en el servicio de urgencias con respecto a los pautados posteriormente durante el ingreso, así como la realización de un análisis comparativo y coste-efectivo con respecto a las plantas de hospitalización.

\section{BIBLIOGRAFÍA}

1. Cuxart A, Estrada O. Hospitalización a domicilio: oportunidad para el cambio. Med Clin (Barc). 2012;138(8):355-360. DOI: 10.1016/j.medcli.2011.04.008.

2. Shepperd S, Harwood D, Jenkinson C, Gray A, Vessey M, Morgan P. Randomized controlled trial comparing hospital at home care with inpatient hospital care. I: three month follow up of health outcomes. BMJ. 1998;316:1786-91. PubMed PMID: 9624068.

3. Hermida M, Dopico L, Lamelo F, Aldámiz-Echevarría B, Silva M, Vidán L. Hospitalización a Domicilio en hospitales públicos gallegos. Galicia Clin. 2015; 76(1):7-12.

4. Tudela P, Mòdol J. La saturación en los servicios de urgencias hospitalarios. Emergencias. 2015;27:113-20. 
5. Alonso G, Escudero J. La unidad de corta estancia de urgencias y la hospitalización a domicilio como alternativa a la hospitalización convencional. An. Sist. Sanit. Navar. 2010;33 Suppl 1:97105.

6. Shepperd S, Lannin N, Clemson L, McCluskey A, Cameron I, Barras S. Discharge planning from hospital to home. Cochrane Database of Systematic Reviews 2013, Issue 1. Art. No.: CD000313. DOI: 10.1002/14651858.CD000313.pub4.

7. Mujal A, Solá J, Hernández M, Aragüás C, Machado M, Oristrell J. Eficacia y seguridad del tratamiento antibiótico domiciliario endovenoso en pacientes con patología infecciosa procedentes del servicio de urgencias. Emergencias. 2013;25:31-6.

8. Pérez J, San José A, Alemán C, Pardos-Gea J, Vilardell M. Antibioterapia intravenosa domiciliaria en una unidad de hospitalización a domicilio: factores pronósticos de reingreso hospitalario. Med Clin (Barc). 2008;131(8):290-2. DOI: 10.1016/S0025-7753(08)72260-7.

9. Conley J, O'Brien C, Leff B, Bolen S, Zulman D. Alternative Strategies to Inpatient Hospitalization for Acute Medical Conditions. A Systematic Review. JAMA Intern Med. 2016;176(11):1693-1702. DOI: 10.1001/jamainternmed.2016.5974.

10. Jiménez S, Antolín A, Aguiló S, Sánchez M. Hospitalización a domicilio directamente desde urgencias: una opción posible y eficiente. Med Clin (Barc). 2010;134(2):87-92. DOI: 10.1016/j. medcli.2008.12.031. 